\title{
A case of brain calcifications in postsurgical hypoparathyroidism
}

\author{
Marco Petrarca $^{1}$ (D) Rosa Scipioni $^{1} \cdot$ Paolo Di Giosia $^{1} \cdot$ Paolo Giorgini $^{1}$. \\ Claudio Ferri ${ }^{1}$
}

Received: 19 February 2016/ Accepted: 25 February 2016/Published online: 11 March 2016

(C) SIMI 2016

A 69-year-old woman presented to our inpatient clinic to evaluate a 2-month history of widespread joint pain, muscle cramps, slowness of movements, tremor of the head, and difficulty in speech. She had suffered from arterial hypertension, secondary hypothyroidism, and hypoparathyroidism for 30 years, due to a papillary thyroid cancer for which she had undergone thyroparathyroidectomy and a radioactive iodine therapy (I-131). There was no history of preexisting neuropsychiatric manifestations, mood disturbances or seizure, and her family history was not contributory. Her drug treatment was amlodipine $10 \mathrm{mg} / \mathrm{die}$, oral levo-tiroxin $125 \mu \mathrm{g} / \mathrm{die}$, and calcitriol $0.25 \mu \mathrm{g} / \mathrm{die}$, and she had discontinued the supplemental calcium salts intake for the 2 prior years, because of the findings of joint and kidney calcifications.

At admission, she was conscious and oriented to the time and place, with a normal memory and intelligence. Vital signs were within normal limits, excepting a poor drug control of the blood pressure $(181 / 103 \mathrm{mmHg})$. Neurological examination confirmed the intentional tremors of the head and the upper limbs, a gait disorder with mild impairment of balance and substantially normal coordination, and dysarthric speech with "punctuated voice". Otherwise, she had neither motor weakness nor cranial nerve involvement, presented only mild bradykinesia without rigidity or stooped posture, and absent

Marco Petrarca

marco.petrarca@hotmail.it

1 University of L'Aquila, Department of Life, Health and Environmental Sciences, Division of Internal Medicine and Nephrology, San Salvatore Hospital, Delta 6 Building, Viale San Salvatore, 67100 Coppito (L'Aquila), Italy primitive reflexes. Her remaining clinical examinations were unremarkable.

Routine laboratory tests revealed normal hemogram, renal and liver function, serum electrolytes such as sodium, potassium, chloride and magnesium, protidogram, and standard urinalysis. Laboratory investigations of thyroid and parathyroid function and calcium-phosphorus metabolism showed hypocalcemia (total calcium $6.7 \mathrm{mg} / \mathrm{dL}$, ionized calcium $3.03 \mathrm{mg} / \mathrm{dL}$ ) and hyperphosphatemia $(4.9 \mathrm{mg} / \mathrm{dL})$, with normal values of thyroid-stimulating hormone (TSH, $0.83 \mu \mathrm{UI} / \mathrm{mL}$ ), free thyroxine (fT4, $1.18 \mathrm{ng} / \mathrm{dL}$ ), parathyroid hormone (PTH, $9.1 \mathrm{pg} / \mathrm{mL}$ ) and vitamin D (22 $\mathrm{ng} / \mathrm{mL})$. Moreover, blood tests for rheumatic diseases such as rheumatoid factor, antinuclear antibody test, anti-cyclic citrullinate peptide antibody and extractable nuclear antigen panel were all unremarkable.

To clarify the neurological involvement, an unenhanced brain magnetic resonance imaging (MRI) was performed, showing homogeneously altered signal intensity in the putamen, globus pallidus, caudate and cerebellar hemispheres (Fig. 1a). To rule out a chronic calcium deposition disease, an unenhanced brain computed tomography (CT) was carried out, confirming diffuse calcified concretions of basal ganglia and cerebellum (Fig. 1b, c). Based on these findings, a diagnosis of Fahr's syndrome was made.

The patient's treatment was implemented with oral calcium and vitamin D3 supplements $1000 \mathrm{mg}+880 \mathrm{UI} /$ die, levodopa and benserazide $100+25 \mathrm{mg} / \mathrm{die}$, and alprazolam $0.5 \mathrm{mg} / \mathrm{die}$, observing an improvement in neurological signs and symptoms.

Bilateral striatopallidodentate calcinosis (BSPDC), otherwise known as Fahr's syndrome, is a rare neurodegenerative condition characterized by symmetric, nonatherosclerotic abnormal calcification of the areas of the 

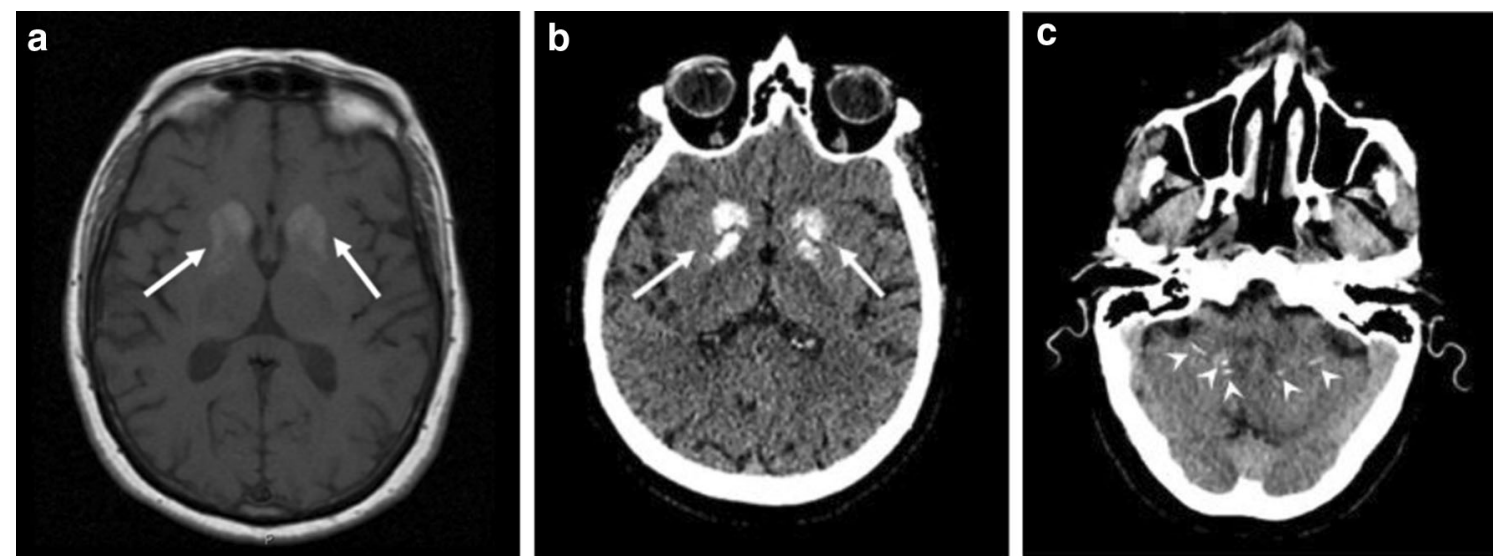

Fig. 1 Unenhanced brain magnetic resonance imaging (a) shows homogeneously altered signal intensity in putamen, globus pallidus, and caudate (arrows). Unenhanced computed tomography reveals diffuse calcifications of basal ganglia (b, arrows) and cerebellar hemispheres (c, arrowheads)

conventional MRI sequences; therefore, a CT scan is generally the favorite imaging technique for detecting and localizing the extent of intracranial calcifications. Also, in cases of suspected BSPDC with brain calcifications on MRI, a CT scan should be considered for a more accurate diagnosis $[1,5]$.

To date, various treatments have been proposed for the management of BSPDC to obtain remission or stabilization of signs and symptoms. Although postsurgical hypoparathyroidism is a well-known condition, it is mandatory to take into account a proper dosage of the supplementary therapy with calcium and vitamin D, to obtain a correct balance between calcium and phosphorus and to avoid systemic calcium deposits. In the same way, movement disorders related to parathyroid diseases can be resolved with the correction of calcium and phosphate. Moreover, treatment includes symptomatic support such as levodopa, clonazepam, and atypical antipsychotics to treat extrapyramidal and psychiatric disorders $[1,3]$.

In conclusion, this report concerns a neurological involvement during a postsurgical hypoparathyroidism not well controlled by drugs. This condition requires a long-term follow-up based on clinical and biochemical data, since hypocalcemia may remain subclinical for an indefinite period of time. Nevertheless, it is essential to take into account the development of intracranial calcifications during this phase, which may be at least partially responsible for neurological symptoms. In this sense, it is worthy considering the use of imaging techniques in those cases where complementary brain alterations are suspected. Equally, an adequate treatment with calcium and hydroxy-vitamin $\mathrm{D}$ should be maintained, paying attention to an adequate serum calciumto-phosphorus ratio to avoid the development of brain calcifications. 


\section{Compliance with ethical standards}

Conflict of interest The authors declare that they have no conflict of interest.

Statement of human and animal rights This article does not contain any studies with human participants or animals performed by any of the authors.

Informed consent Informed consent was obtained from the individual participant included in the study.

\section{References}

1. Saleem S, Aslam HM, Anwar M, Anwar S, Saleem M, Saleem A, Rehmani MA (2013) Fahr's syndrome: literature review of current evidence. Orphanet J Rare Dis 8(8):156
2. Geschwind DH, Loginov M, Stern JM (1999) Identification of a locus on chromosome 14q for idiopathic basal ganglia calcification (Fahr disease). Am J Hum Genet 65(3):764-772

3. Asokan AG, D'souza S, Jeganathan J, Pai S (2013) Fahr's syndrome - an interesting case presentation. J Clin Diagn Res 7(3):532-533

4. Manyam BV, Walters AS, Narla KR (2001) Bilateral striopallidodentate calcinosis: clinical characteristics of patients seen in a registry. Mov Disord 16(2):258-264

5. Avrahami E, Cohn DF, Feibel M, Tadmor R (1994) MRI demonstration and CT correlation of the brain in patients with idiopathic brain intracerebral calcification. $\mathrm{J}$ Neurol 241(6):381-384 